\title{
Paleopalinología y fechamiento por U-Pb en zircones de la Formación El Cien (Oligoceno), Baja California Sur, México
}

\author{
Paleopalynology and U-Pb zircon dating of the El Cien Formation (Oligocene), Baja California \\ Sur, Mexico
}

Iris G. Galván-Escobedo ${ }^{1}$, Elia Ramírez-Arriaga ${ }^{2}$, Alfonso Valiente-Banuet ${ }^{3}$, Ebandro Uscanga-Mortera ${ }^{1 *}$, Edmundo García-Moya ${ }^{1}$, Josué Kohashi-Shibata ${ }^{1}$

\begin{abstract}
Posgrado en Botánica, Colegio de Postgraduados, Campus Montecillo, Carretera México-Texcoco, Km. 36.5, Montecillo, Texcoco, 56230, Estado de México, México.

${ }^{2}$ Laboratorio de Palinología, Departamento. de Paleontología, Instituto de Geología, Universidad Nacional Autónoma de México, Ciudad Universitaria, 04510, Coyoacán, CDMX, México.
\end{abstract}

${ }^{3}$ Departamento de Ecología de la Biodiversidad, Instituto de Ecología, Universidad Nacional Autónoma de México, Ciudad Universitaria, 04510, Coyoacán, CDMX, México.

* Autor para correspondencia: (E. UscanaMortera)euscanga@colpos.mx

\begin{abstract}
Cómo citar este artículo:
Galván-Escobedo, I.G., Ramírez-Arriaga, E., Valiente-Banuet, A., Uscanga-Mortera, E., García-Moya, E., Kohashi-Shibata, J., 2020, Paleopalinología y fechamiento por $\mathrm{U}-\mathrm{Pb}$ en zircones de la Formación El Cien (Oligoceno), Baja California Sur, México: Boletín de la Sociedad Geológica Mexicana, 72(2), A300819. http://dx.doi.org/10.18268/ BSGM2020v72n2a300819
\end{abstract}

Manuscrito recibido: 20 de Mayo de 2019 Manuscrito corregido: 6 de Julio de 2019 Manuscrito aceptado: 23 de Agosto de 2019

La revisión por pares es responsabilidad de la Universidad Nacional Autónoma de México.

Estees un artículo Open Accessbajo la licencia CCBY-NC-SA (https://creativecommons.org/licenses/by-nc-sa/4.0/)

\section{RESUMEN}

En las cercanías de San Juan de la Costa, Baja California Sur, se extrajo un barreno (B187) cuyos estratos geológicos corresponden a la Formación El Cien. El fechamiento por el método U-Pb en Zircones indicó que las rocas que conforman dicho barreno se depositaron hace aproximadamente 27.5 millones de años (Oligoceno superior). El estudio paleopalinológico del B187 mostró que en estos estratos se conservan microfósiles (palinomorfos) de origen continental (polen y esporas) y marinos (quistes de dinoflagelados), los cuales fueron cuantificados e identificados. El conjunto total de palinomorfos tuvo una riqueza de 248 taxones, 230 correspondientes a formas continentales y 18 a dinoflagelados. El índice de diversidad a de especies del conjunto total fue de $H^{\prime}=3.3$, con una uniformidad de $\mathcal{f}^{\prime}=0.6$; los valores de diversidad para el conjunto continental y el de dinoflagelados fueron de $H^{\prime}=3.6, \mathcal{J}^{\prime}=0.7$ y $H^{\prime}=1.8, \mathcal{J}^{\prime}=0.6$, respectivamente. Un análisis de agrupamiento CONISS definió tres palinozonas en la columna estratigráfica del barreno: 1) la palinozona A, que se caracterizó por una pobre riqueza de polen-esporas y dinoflagelados; 2) la palinozona B, que sobresalió por concentrar la mayor riqueza y diversidad de palinomorfos continentales y dinoflagelados, y 3) la palinozona $\mathrm{C}$, que se distinguió por ser la menos productiva en cuanto palinomorfos. Los taxones que dominaron el conjunto continental se asociaron con la presencia de bosque tropical caducifolio tales como Chenopodipollis (Amaranthaceae-Chenopodiaceae), Graminidites (Poaceae), Psilatricolpites (Leguminosae), Liliacidites (Liliaceae, Bromeliaceae y Palmae) y el tipo Stercualiaceae. Por otro lado, algunos otros taxones se consideraron como elementos alóctonos representantes del bosque mesófilo de montaña, como el polen de Eucommia, Fraxinoipollenites (Fraxinus), Betulaceae, Jungladaceae, y diversas esporas entre las que destacó Lusatisporis dettmannae (Selaginella oregana). En el conjunto de dinoflagelados sobresalieron por su abundancia los géneros de Operculodinium y Spiniferites ramosus.

Palabras clave: palinomorfos continentales y marinos, diversidad de especies, bosque tropical caducifolio, bosque mesófilo de montaña, comparaciones palinoflorísticas.

\section{ABSTRACT}

Borehole (B187) near San Fuan de la Costa, Baja California Sur, was collected. The strata of this borehole consists of the El Cien Formation. Dating by $\mathrm{U}-\mathrm{Pb}$ method in Zircons indicated that the sediments were deposited 27.5 million years ago (upper Oligocene). The B187 paleopalynological research showes that in these strata, palynomorphs of both continental (pollen and spores) and marine (dinoflagellate cysts) origin are preserved, which were quantified and identified. The palynomorphs' richness consists of 248 taxa, 230 were continental and 18 dinoflagellates. The a diversity index of total palinomorphs was $H^{\prime}=3.3$, with uniformity $\bar{y}^{\prime}=0.6$. Values of a diversity for continental and dinoflagellates groups were $H^{\prime}=$ 3.6, $\mathcal{J}^{\prime}=0.7$ and $H^{\prime}=1.8, \mathcal{J}^{\prime}=0.6$, respectively. The CONISS cluster analysis defined three palynozones throughout the borehole stratigraphic column: palynozone A, distinguished by a poor richness of both, pollen-spores and dinoflagellates; in contrast, palynozone B concentrated the highest richness and diversity of continental palinomorphs and dinoflagellates; and palynozone $C$, had the lowest palinomorphs richness. The assemblage of continental microfossils were dominated by Chenopodipollis (Amaranthaceae-Chenopodiaceae), Graminidites (Poaceae), Psilatricolpites (Leguminosae), Liliacidites (Liliaceae, Bromeliaceae y Palmae) and the Stercualiaceae type, all of which are currently associated with the presence of tropical deciduous forest. On the other hand, pollen of Eucommia, Fraxinoipollenites (Fraxinus), Betulaceae, Fungladaceae, are all allochthonous elements belonging to tropical mountain cloud forests, and diverse spores highlighted by Lusatisporis dettmannae (Selaginella oregana). In the dinoflagellates assemblage, both Operculodinium and Spiniferites ramosus abundance.

Keyzords: continental and marine palynomorphs, alpha diversity, tropical deciduous forest, tropical mountain cloud forests, palynomorphic comparisons. 


\section{Introducción}

En el Cretácico (hace 66 millones de años $-\mathrm{Ma}^{-}$) la posición del extremo sur de la península de Baja California se encontraba en Cabo Corrientes, cerca de Puerto Vallarta, Jalisco (Smith, 1991; Ledesma-Vázquez y Carreño, 2010). Los datos paleontológicos y estratigráficos indican que esta condición se mantuvo durante el Oligoceno al Mioceno (hace 33.9 a $5.3 \mathrm{Ma}$ ) (Aranda-Gómez et al., 2000; Carreño y Helenes, 2002). De esta manera, durante el Paleógeno/Neógeno (66 a 2.6 Ma), gran parte de Baja California Sur (BCS) estuvo cubierto por un mar somero en el cual se depositaron más de $4000 \mathrm{~m}$ de sedimentos (Alatorre, 1988). Esto originó una secuencia estratigráfica de sedimentos en la región, que dieron origen a las formaciones geológicas: Tepetate (Paleoceno-Eoceno), San Gregorio (en el área de La Purísima) (Oligoceno superior-Mioceno inferior), El Cien (en el área de San Juan de la Costa) (Oligoceno superior), San Isidro (Mioceno inferior o medio), Comondú (Oligoceno superior-Mioceno medio) y La Salada (Plioceno) (Hausback, 1984; Applegate, 1986; Alatorre, 1988; Fischer et al., 1995; Carreño y Smith, 2007) (Figura 1).

La geología de la Formación El Cien (FEG) fue descrita y nombrada por Applegate (1986) en los alrededores del poblado El Cien, en el municipio de La Paz, BCS. Investigaciones posteriores llevadas a cabo por Fischer et al. (1995) determinaron que las rocas de la FEG afloran en dos cinturones continuos a los lados de la Sierra La Giganta, los cuales forman un sinclinal somero. En el oeste, dicha Formación aflora en el valle del arroyo Aguajito, y en el este, a lo largo de la costa en la localidad San Juan de la Costa. Litológicamente se pueden diferenciar dos miembros en ella: el miembro basal denominado miembro San Fuan y el superior, el miembro Cerro Colorado.

De acuerdo con la evidencia bioestratigráfica y radiométrica (Hausback, 1984; Kim y Barron, 1986), la edad de la FEC es Oligoceno superior-Mioceno inferior (28.1 a 21 Ma).De manera general, los sedimentos depositados durante el Oligoceno-Mioceno, en BCS, han sido poco estudiados desde el punto de vista paleopalinológico (Galván-Escobedo et al., 2012; Galván-Escobedo et al., 2013; Galván-Escobedo et al., 2015; Galván-Escobedo et al., 2017), especialmente aquellos procedentes de la FEG. La recuperación de palinomorfos fósiles a partir de los sedimentos correspondientes a la FEC, puede ser de gran interés para entender la antigüedad y la distribución de diferentes organismos vegetales a través del tiempo (Rzedowski, 2006). En particular, el polen fósil puede aportar información valiosa que contribuya al estudio de los procesos evolutivos de la flora y la vegetación de la región y de México.En este trabajo se presentan los resultados paleopalinológicos obtenidos del estudio del barreno 187 correspondiente a la FEC. Se analiza la riqueza y diversidad de especies a de los conjuntos paleopalinológicos, dando mayor énfasis al conjunto de microfósiles continentales (polen y esporas de pteridofitas) y, con base en ellos, se proponen los tipos de vegetación que se desarrollaron durante el Oligoceno superior en la zona de San Juan de la Costa, BCS.

\begin{tabular}{|c|c|c|c|c|c|c|c|}
\hline 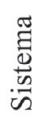 & $\begin{array}{l}\frac{0}{0} \\
\frac{0}{10} \\
0\end{array}$ & \multicolumn{2}{|l|}{ Época } & Etapa & 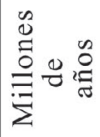 & \multicolumn{2}{|r|}{$\begin{array}{c}\text { Fisher et al., } \\
1995 \\
\text { SJC }\end{array}$} \\
\hline \multirow{18}{*}{$\frac{0}{\frac{\pi}{0}}$} & \multirow{9}{*}{ 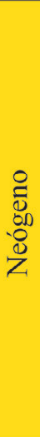 } & \multirow{3}{*}{ Plioceno } & \multirow{2}{*}{$\mathrm{U}$} & Gelasiense & 2.6 & \multirow{3}{*}{\multicolumn{2}{|c|}{$?$}} \\
\hline & & & & Piacenziense & 3.6 & & \\
\hline & & & $\mathrm{L}$ & Zancliense & 5.3 & & \\
\hline & & \multirow{6}{*}{ Mioceno } & \multirow{2}{*}{$\mathrm{U}$} & Messiniense & 7.2 & \multirow{4}{*}{\multicolumn{2}{|c|}{ Comondú }} \\
\hline & & & & Tortoniense & 11.6 & & \\
\hline & & & \multirow{2}{*}{ M } & Serravalliense & 13.8 & & \\
\hline & & & & Langhiense & 16.0 & & \\
\hline & & & \multirow{2}{*}{$\mathrm{L}$} & Burdigaliense & 20.4 & \multirow{3}{*}{$\frac{\stackrel{\Xi}{U}}{\stackrel{\Xi}{ \pm}}$} & \multirow{2}{*}{$\begin{array}{c}\text { Miembro } \\
\text { Cerro Colorado }\end{array}$} \\
\hline & & & & Aquitaniense & $23.0 *$ & & \\
\hline & \multirow{9}{*}{$\begin{array}{l}0 \\
\stackrel{1}{0} \\
\infty \\
0 \\
\frac{0}{\pi} \\
0\end{array}$} & Oligacenn & $\mathrm{U}$ & Chattiense & $28.1 *$ & & $\begin{array}{l}\text { Miembro } \\
\text { San Juan }\end{array}$ \\
\hline & & Oingüerio & L & Rupeliense & 33.9 & & \\
\hline & & \multirow{4}{*}{ Eoceno } & $\mathrm{U}$ & Priaboniense & 37.8 & \multirow{7}{*}{\multicolumn{2}{|c|}{ Tepetate }} \\
\hline & & & \multirow{2}{*}{ M } & Bartoniense & 41.2 & & \\
\hline & & & & Luteciense & 47.8 & & \\
\hline & & & $\mathrm{L}$ & Ypresiense & 56.0 & & \\
\hline & & & $\mathrm{U}$ & Thanetiense & 59.2 & & \\
\hline & & Paleoceno & M & Selandiense & 61.6 & & \\
\hline & & & $\mathrm{L}$ & Daniense & 66 & & \\
\hline
\end{tabular}

Figura 1 Secuencia estratigráfica de las formaciones geológicas en la zona de San Juan de la Costa, Baja California Sur, México. 


\section{Métodos}

\subsection{LOGALIZAGIÓN DEL ÁREA DE ESTUDIO}

El barreno 187 (B187) fue extraído por el personal de la empresa minera Roca Fosfórica Mexicana (ROFOMEX), ubicada en San Juan de la Costa, La Paz, BCS. Las coordenadas geográficas del punto de extracción del B187 son 24²7’5.8” N $110^{\circ} 43^{\prime} 56.2^{\prime \prime} \mathrm{W}$ (Figura 2).

\subsection{MUESTREO Y TRATAMIENTO DE LAS MUESTRAS}

El B187 tuvo una profundidad de 103.90 m. Está conformado por arenisca tobácea y calcárea de grano fino a grueso, entre la cual se encuentran intercalaciones de lutita. En la parte media se localizan nódulos calcáreos que son característicos del miembro San Juan (Figura 3).

Se observó la litología del B187 y se tomó una muestra de roca cada vez que se notó un cambio de color y textura. La muestra Pb-10199, conformada por arenisca tobácea, se utilizó para fechar el barreno por el método U-Pb (Uranio-Plomo) en zircones (Solari et al., 2010). Los zircones los separó la Dra. Consuelo Macías del Instituto de Geología de la UNAM (IG-UNAM), y el fechamiento lo realizó el Dr. Luigui Solari del Laboratorio de Estudios Isotópicos del Centro de Geociencias de la UNAM.

Se obtuvo un total de 69 muestras (Figura 3), las cuales se llevaron al laboratorio de Paleopalinología del IG-UNAM para la extracción de palinomorfos. Para dicho propósito se usaron métodos estándares (ácido clorhídrico, ácido fluorhídrico, ácido nítrico, acetólisis y flotación con politungstato de sodio). Se elaboraron laminillas permanentes con hidroxiacetil celulosa y bálsamo de Canadá, las cuales se depositaron en la colección de polen fósil del IG-UNAM. Se revisó una laminilla de cada muestra en un microscopio óptico Zeiss, con objetivo 100x y contraste de fases. En cada laminilla, una sola persona contó todos los palinomorfos; la productividad de cada muestra varió entre 4 y 5914 palinomorfos. Se tomaron fotomicrografías con una cámara
AxioCam ICc1-Zeiss y el programa AxioVision versión 4.8.2.

\subsection{ANÁLISIS DE LA INFORMAGIÓN}

Los palinomorfos registrados se agruparon en dos conjuntos: 1) el continental, que incluye el polen y las esporas; y 2) el marino, representado por los quistes de dinoflagelados (referidos como dinoflagelados), acritarcas y prasinofitas, huevos de copépodo, escolecodontos y testas de microforaminíferos. Ambos conjuntos se tomaron como base para la aplicación de los análisis de diversidad de especies y de distribución y agrupamiento de los taxones (palinozonas). En los análisis efectuados específicamente sobre el conjunto marino, se excluyeron los grupos sobrerrepresentados (las acritarcas/prasinofitas, huevos de copépodos, escolecodontos y testas de microforaminíferos,) quedando solamente los dinoflagelados. Posteriormente, con énfasis en el conjunto continental, se llevó a cabo un análisis de los tipos de vegetación.

\subsubsection{DIVERSIDAD DE ESPECIES}

Para contrastar la diversidad de taxones entre el conjunto continental y el de dinoflagelados, se calculó la riqueza de especies; la diversidad a de especies, mediante el índice de diversidad de Shannon-Wiener $\left(H^{\prime}\right)$ con base en el logaritmo natural; y su uniformidad (f') asociada (Pielou, 1975; Magurran, 1989). Para contrastar los valores de diversidad entre muestras, dichos cálculos se realizaron de manera conjunta sobre el total de los elementos continentales y los dinoflagelados. Los cálculos y estimaciones se realizaron con el programa Species Diversity and Richness IV (Seaby y Henderson, 2007).

\subsubsection{DISTRIBUCIÓN DE TAXONES Y PALINOZONAS EN LA COLUMNA ESTRATIGRÁFICA}

Con la finalidad de observar la distribución de los taxones registrados en cada una de las muestras, se elaboraron diagramas polínicos con las frecuencias absolutas y porcentajes. Con base en 
las frecuencias absolutas, se aplicó un análisis de agrupamiento CONISS (constrained incremental sum of squares clustering) (Grimm, 1987) que definió las palinozonas en la columna estratigráfica del B187. Los diagramas polínicos y el CONISS se realizaron en el programa Tilia versión 1.7.16 (Grimm, 2011).

\subsubsection{PALEOVEGETACIÓN}

El conjunto paleopalinológico continental aportó información sobre los posibles tipos de vegetación que pudieron desarrollarse durante el Oligoceno superior en la región de San Juan de la Costa. Los tipos de vegetación se definieron con base en la frecuencia absoluta de los taxones de polen y esporas fósiles que fueron identificados a nivel de forma-género fósil o por su afinidad con especies actuales; los taxones no determinados se suprimieron para ejecutar el análisis. Para lograr este objetivo, se realizó un análisis de agrupamiento jerárquico usando el método Ward y una distancia euclidiana de 120 (Johnson, 2000). Los cálculos se ejecutaron con el software PAST versión 3.0.
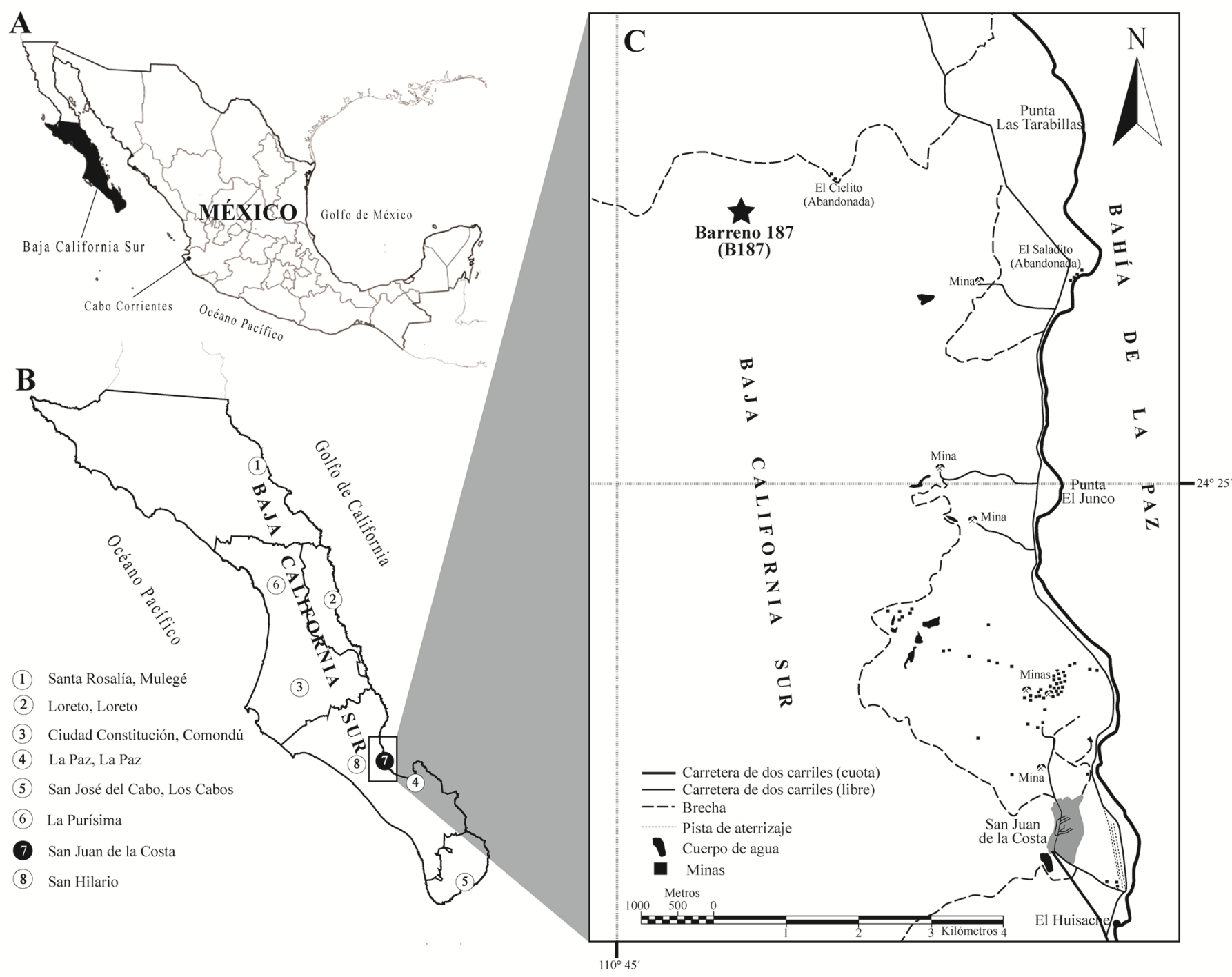

Figura 2 Localización geográfica del sitio de estudio. A) Ubicación del estado de Baja California Sur. B) Localización de la comunidad de San Juan de la Costa. C) Punto de extracción del barreno 187. 


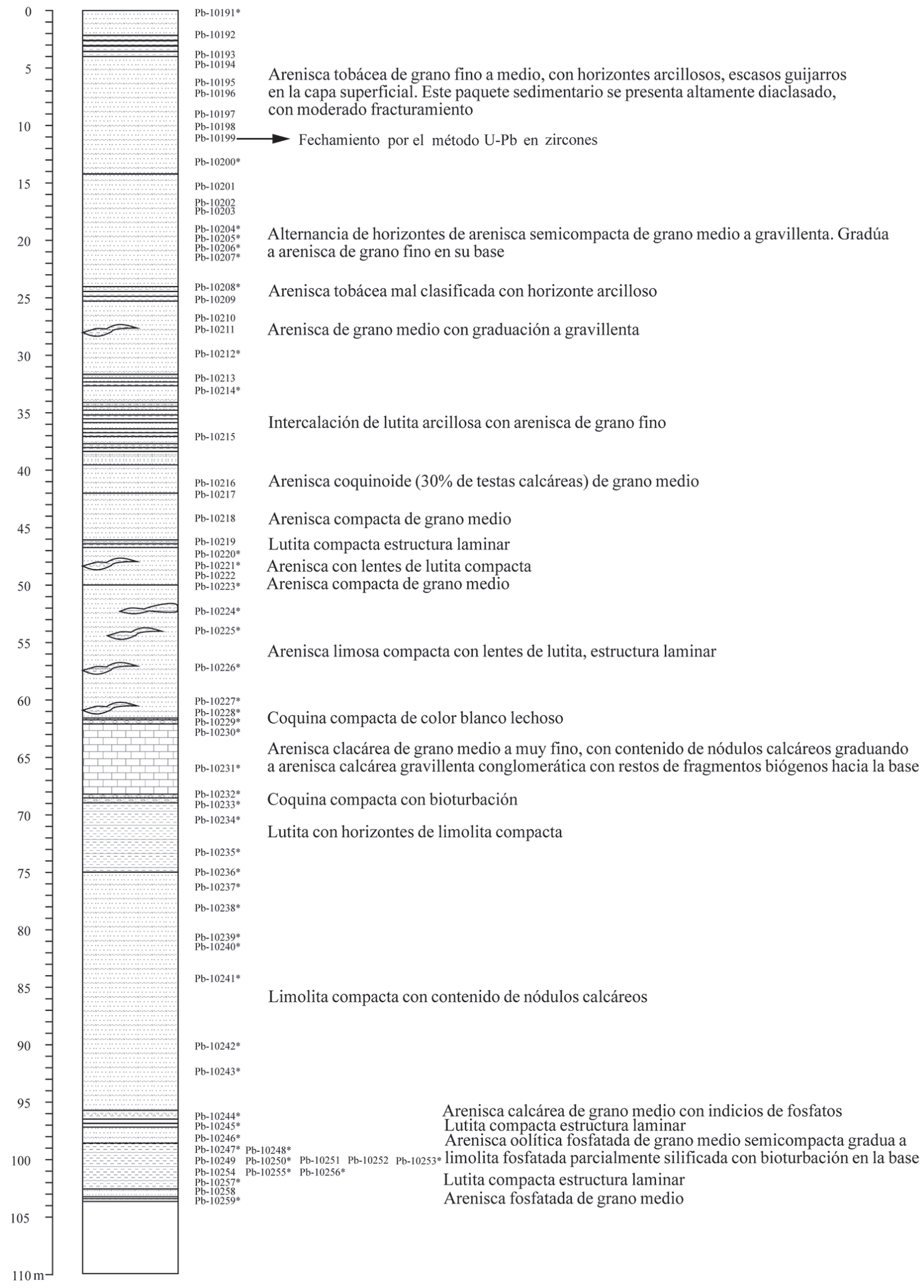




\section{Resultados}

\subsection{FEGHAMIENTO POR EL MÉTODO U-PB EN ZIRCONES}

Los Zircones se localizaron en la cima de la columna estratigráfica del B187 (Pb-10199). El fechamiento arrojó una edad de $27.50 \mathrm{Ma}+0.40$ -0.70, por lo tanto se puede considerar que los sedimentos del B187 se depositaron durante el Oligoceno superior (Figura 4).

\subsection{DIVERSIDAD DE ESPEGIES}

De las 69 muestras estudiadas, en 41 de ellas se registraron palinomorfos (Tabla 1). Se contaron un total de 64425 palinomorfos. De estos, el 95\% se clasificaron dentro del conjunto marino (dinoflagelados, acritarcas/prasinofitas, huevos de copépodo, escolecodontos y testas de microforaminíferos); el 4\% en el conjunto continental (polen y esporas) y el $1 \%$, fueron fungosporas (Figura 5).

La riqueza total del conjunto paleopalinológico fue de 248 taxones. El mayor número de taxones por muestra fue de $59(\mathrm{~Pb}-10228)$ y el menor fue de 1 (Pb-10212 y Pb-10214). La diversidad a de especies $\left(H^{\prime}\right)$ de todo el conjunto fue de 3.3, con una variación por muestra desde 0.1 (Pb-10212 y $\mathrm{Pb}-10214)$ y hasta $3.3(\mathrm{~Pb}-10241)$, mientras, la uniformidad $\left(\mathcal{J}^{\prime}\right)$ fue de 0.6 en total, y por muestra osciló entre 0 (Pb-10212 y $\mathrm{Pb}-10214)$ y 1 (Pb-10200, Pb-10206, Pb-10207, Pb-10248, $\mathrm{Pb}-10253$ y $\mathrm{Pb}-10255)$.

En el conjunto continental se registraron 2319 palinomorfos, de los cuales el $4 \%$ correspondieron a esporas de pteridofitas, el $26 \%$ a gimnospermas y $70 \%$ a angiospermas. De estas últimas el $85 \%$ fueron dicotiledóneas y el 15\% monocotiledóneas.

La riqueza total de formas continentales fue de 230. La mayor riqueza de taxones por muestra fue de $50(\mathrm{~Pb}-10228)$ y la menor fue de $1(\mathrm{~Pb}-$ 10212, Pb-10214, Pb-10255). La diversidad a de especies $\left(H^{\prime}\right)$ del conjunto continental fue de 3.6, y por muestra obtuvo valores de entre $0(\mathrm{~Pb}-10212$, Pb-10214, Pb-10255) y 3.2 (Pb-10240, Pb-10241), mientras que la uniformidad $\left(\mathcal{J}^{\prime}\right)$ fue de 0.7 en total, y por muestra osciló entre 0 (Pb-10212, Pb-10214, Pb-10255) y 1 (Pb-10200, Pb-10206, Pb-10207, Pb-10231, Pb-10248, Pb-10253).

Por otro lado, en el conjunto marino, los elementos mejor representados fueron las acritarcas y prasinofitas, los cuales representaron el $91 \%$ de este conjunto; por su parte, los huevos de copépodos, los escolecodontos y testas de microforaminíferos el $4 \%$, y los dinoflagelados el 5\%. De este último grupo se contabilizaron 2911 elementos en total.

En particular, la riqueza total del conjunto de dinoflagelados fue de 18 taxones, mientras que por muestra se registraron desde 1 (Pb-10250, $\mathrm{Pb}-10253, \mathrm{~Pb}-10255$ y $\mathrm{Pb}-10257)$ hasta 9 taxones (Pb-10228). La diversidad a de especies $\left(H^{\prime}\right)$ del conjunto de dinoflagelados fue de 1.8, y por muestra obtuvo valores de entre 0 (Pb-10191, Pb-10200, Pb-10205, Pb-10206, Pb-10207, Pb-10212， Pb-10214， Pb-10223， Pb-10234, Pb-10247， Pb-10248， Pb-10250， Pb-10253, $\mathrm{Pb}-10255$ y $\mathrm{Pb}-10257)$ y 1.5 (Pb-10256). Por otro lado, la uniformidad $\left(\mathcal{f}^{\prime}\right)$ fue de 0.6 en total, y por muestra varió entre 0 (Pb-10191, $\mathrm{Pb}-10200$, Pb-10205, Pb-10206, Pb-10207, Pb-10212, Pb-10214, Pb-10223, Pb-10234, Pb-10247, Pb-10248, Pb-10250, Pb-10253, Pb-10255 у $\mathrm{Pb}-10257)$ y 1 (Pb-10220).

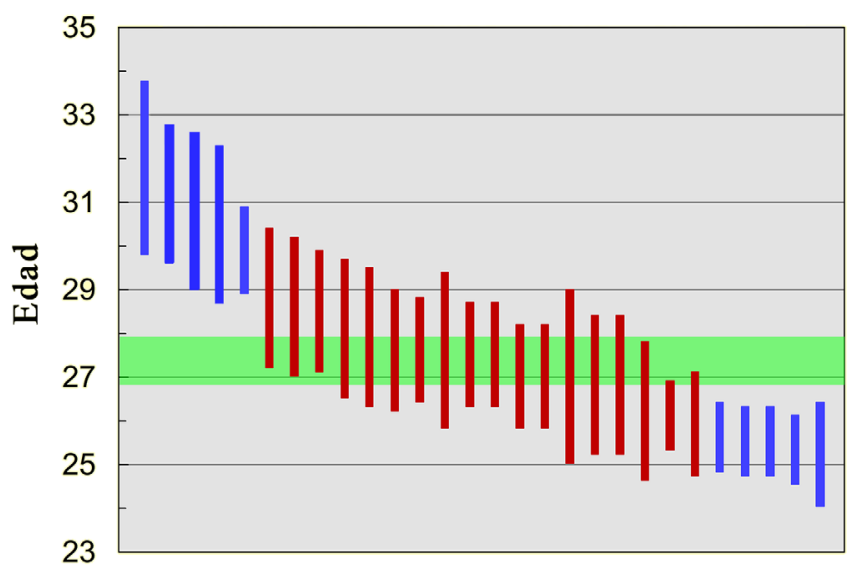

Figura 4 Resultados del fechamiento del barreno 187 por el método $\mathrm{U}-\mathrm{Pb}$ en Zircones. Edad $=27-50+0.40-0.70 \mathrm{Ma}$. Confiabilidad del 96.9 \% basado en un grupo coherente de 18 zircones. 
Tabla 1. Frecuencias absolutas de los palinomorfos continentales y marinos cuantificados en 41 muestras del barreno 187, San Juan de la Costa, Baja California Sur, México.

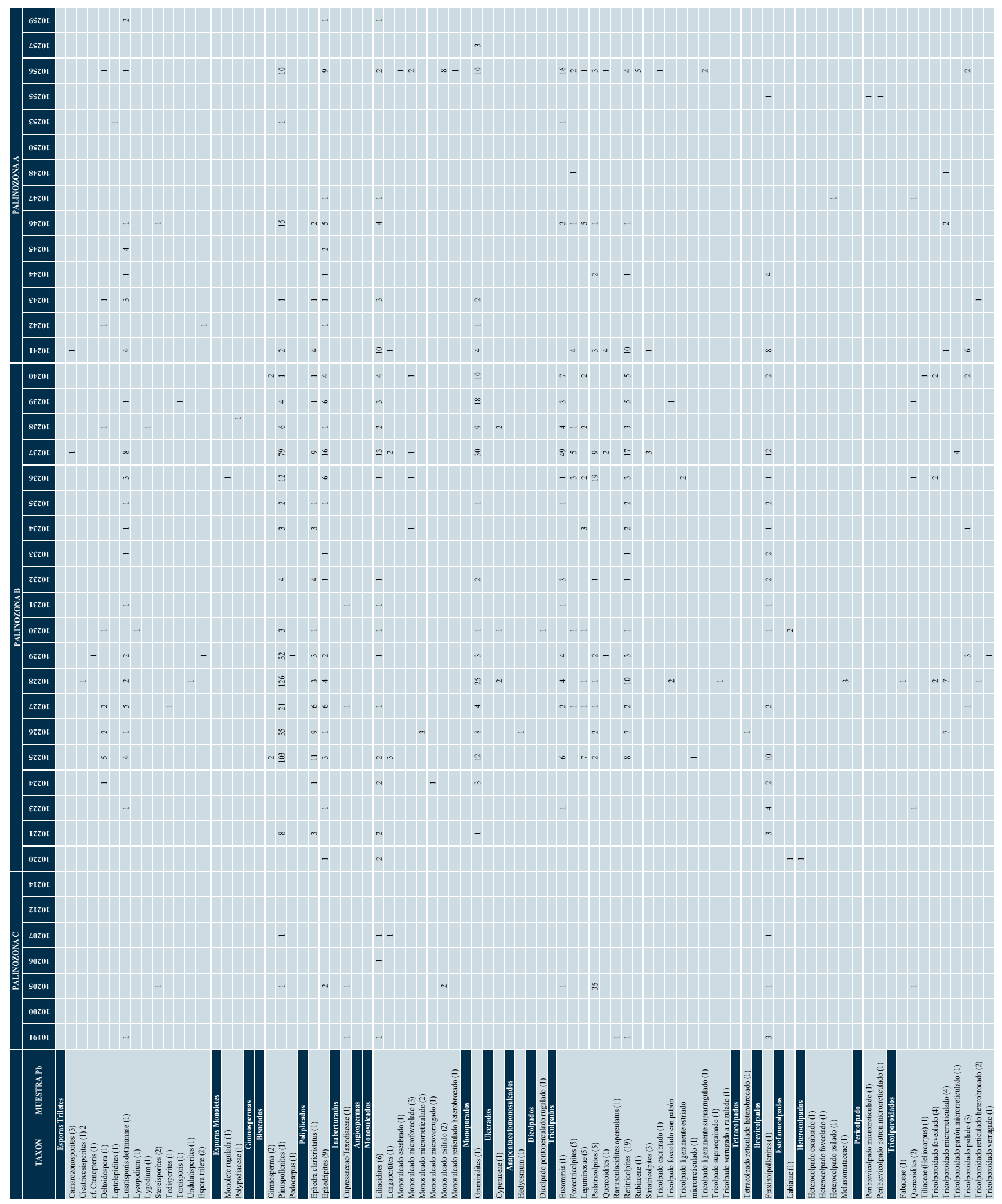


Tabla 1. (Continuación) Frecuencias absolutas de los palinomorfos continentales y marinos cuantificados en 41 muestras del barreno 187, San Juan de la Costa, Baja California Sur, México.

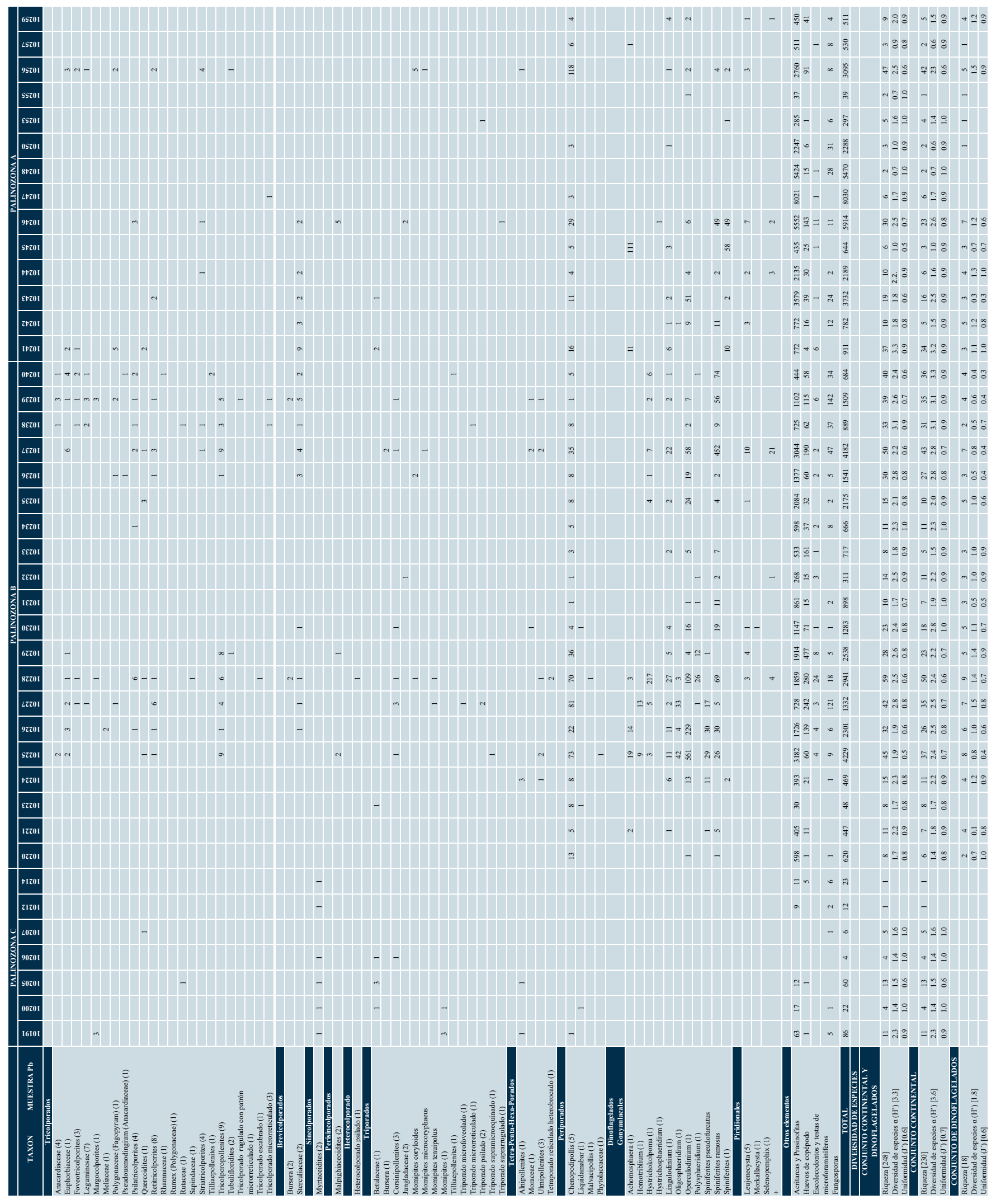


Los valores de los análisis de diversidad de especies del conjunto total, así como de manera independiente de los elementos continentales y los dinoflagelados, pueden observarse en la Figura 6.

\subsection{DISTRIBUGIÓN DE TAXONES Y PALINOZONAS EN LA GOLUMNA ESTRATIGRÁFICA}

El conjunto paleopalinológico del B187 estuvo caracterizado por la presencia de formas continentales como Ephedripites, Ephedra claricristatus, Pinuspollenites, Graminidites, Liliacidites, Corsinipollenites, Chenopodipollis, Eucommia, Fraxinoipollenites, Psilatricolpites, Retitricolpites, Tricolporopollenites y los tipos Anacardiaceae y Sterculiaceae; así como por la presencia de dinoflagelados, tales como Lingulodinium, Oligosphaeridium, Operculodinium, Polysphaeridium, Spiniferites, S. pseudofurcatus, S. ramosus y Leujenecysta. La distribución de las frecuencias absolutas y porcentajes de cada uno de los taxones registrados se presentan en las Figuras 7 y 8 . Algunos de los palinomorfos registrados se muestran en la Figura 9.

Por otro lado, el análisis de agrupamiento CONISS definió tres palinozonas a lo largo de la columna estratigráfica del B187 (Figuras 7 y 8), las cuales fueron nombradas como palinozonas A, B y C:

1. La palinozona A comprende la parte basal del barreno de las muestras Pb-10241 a la $\mathrm{Pb}-10259$. En dicha palinozona se registraron, en general, bajas frecuencias de elementos continentales y marinos. En el conjunto continental, los elementos que mejor se encontraron representados fueron Pinuspollenites, Ephedripites, Liliacidites, Graminidites, Eucommia, Chenopodipollis, y los tipos Leguminosae y Sterculiaceae; mientras que en el conjunto de dinoflagelados sobresalieron: Achomosphaera, Lingulodinium, Spiniferites y Leujenecysta.

2. La palinozona $\mathrm{B}$ abarca la parte intermedia del B187, de las muestras Pb-10220 a la $\mathrm{Pb}-10240$. En esta palinozona se concentró la mayor riqueza de taxones continentales y de dinoflagelados. Los taxones continentales que registraron las mayores frecuencias fueron: Deltoidospora, Lusatisporis dettmannae, Ephedripites, Ephedra claricristatus, Pinuspollenites, Graminidites, Liliacidites, Corsinipollenites, Chenopodipollis Eucommia, Fraxinoipollenites, Pseudousmodingium, Psilatricolpites, Retitricolpites, Tricolporopollenites y los tipos Anacardiaceae, Euphorbiaceae, Leguminosae y Sterculiaceae. Por otro lado, la mayoría de los taxones de dinoflagelados se registraron en esta palinozona, entre ellos se encontraron Achomosphaera, Homotriblium, Hystrichokolpoma, Hystrichosphaeridium, Lingulodinium, Oligosphaeridium, Operculodinium, Polysphaeridium, Spiniferites pseudofurcatus, S. ramosus, Leujenecysta y Selenopemphix.

3. Finalmente, la palizona $\mathrm{C}$ está localizada en la cima del barreno y comprende de las muestras Pb-10191 a Pb-10240. En esta palinozona casi no se registraron palinomorfos. Los únicos taxones continentales existentes se encontraron en bajas frecuencias y fueron Ephedripites, Psilatricolpites, Fraxinoipollenites, tipo Leguminosae, Psilatricolporites, Rumex, tipo Betulaceae, Corsinipollenites, Momipites, Alnipollenites y Liquidambar. No se registraron dinoflagelados en esta palinozona.

\subsection{PALEOVEGETAGIÓN}

En el dendrograma de la Figura 10 se observa que el análisis de agrupamiento jerárquico de los elementos continentales diferenció dos grupos principales, el A y el B.

El grupo A estuvo conformado por los taxones más abundantes del conjunto: Chenopodipollis ( $\mathrm{n}=595)$ y Pinupollenites $(\mathrm{n}=470)$ (Figura 10).

El grupo B se dividió en tres subgrupos definidos como B1, B2, y B3. El subgrupo B1 estuvo constituido por dos taxones que también mostraron altas frecuencias: Gaminidites $(\mathrm{n}=147)$ y Eucommia $(\mathrm{n}=106)$ (Figura 10). El subgrupo B2 fue el grupo con la mayor cantidad de taxones, pero con frecuencias más bajas por taxones ( $\mathrm{n}=1$ a 25). Treinta y dos de los taxones del subgrupo B2 son elementos que actualmente se encuentran en el bosque mesófilo de montaña, 22 de ellos se desarrollan en el bosque tropical caducifolio y cuatro son elementos 
que pueden vivir en ambos tipos de vegetación. En el subgrupo B3 destacó la presencia de elementos del bosque tropical caducifolio como Ephedripites ( $\mathrm{n}=77$ ), Ephedra claricristatus $(\mathrm{n}=63)$, Psilatricolpites $(\mathrm{n}=77)$, Liliacidites $(\mathrm{n}=60)$ y Sterculiaceae $(\mathrm{n}=37)$ (Figura 10).

\section{Discusión}

\subsection{DIVERSIDAD DE ESPEGIES}

El referente más próximo para comparar la diversidad de especies registradas en el B187 de la Formación El Cien (Oligoceno superior) son los datos obtenidos del barreno B4 de la Formación San Gregorio (Oligoceno superior-Mioceno inferior) (Galván-Escobedo et al., 2017), localizado al norte de Baja California Sur.

El B187 fue muy productivo desde el punto de vista paleopalinológico. Se registraron en total 64425 palinomorfos, mientras que en el B4 se contaron 25470. Sin embargo, la proporción de los conjuntos paleopalinológicos continental y marino fue muy similar en los dos barrenos, ya que en ambos el conjunto marino conformó más del $90 \%$, mientras que el continental fue menor al $10 \%$.

La riqueza total de taxones fue considerablemente más alta en el B187 (248), en comparación con la riqueza total del B4 (189). Además, es de destacar que la riqueza de las formas continentales en el B187 fue mayor (230) en contraste con la del B4 (161). De manera inversa ocurrió en el caso del conjunto de dinoflagelados, ya que el B4 registró 28 taxones, mientras que en el B187 solo se encontraron 18.

La diversidad a de especies del conjunto total en el B187 $\left(H^{\prime}=3.3\right)$ fue ligeramente mayor que el caso del B4 $\left(H^{\prime}=3.02\right)$, mientras que la uniformidad no tuvo diferencias significativas en ambos barrenos $\left(\mathrm{B} 187, \mathcal{f}^{\prime}=0.6\right.$; B4, $\left.\mathcal{f}^{\prime}=0.57\right)$. La diversidad a de especies del conjunto de microfósiles

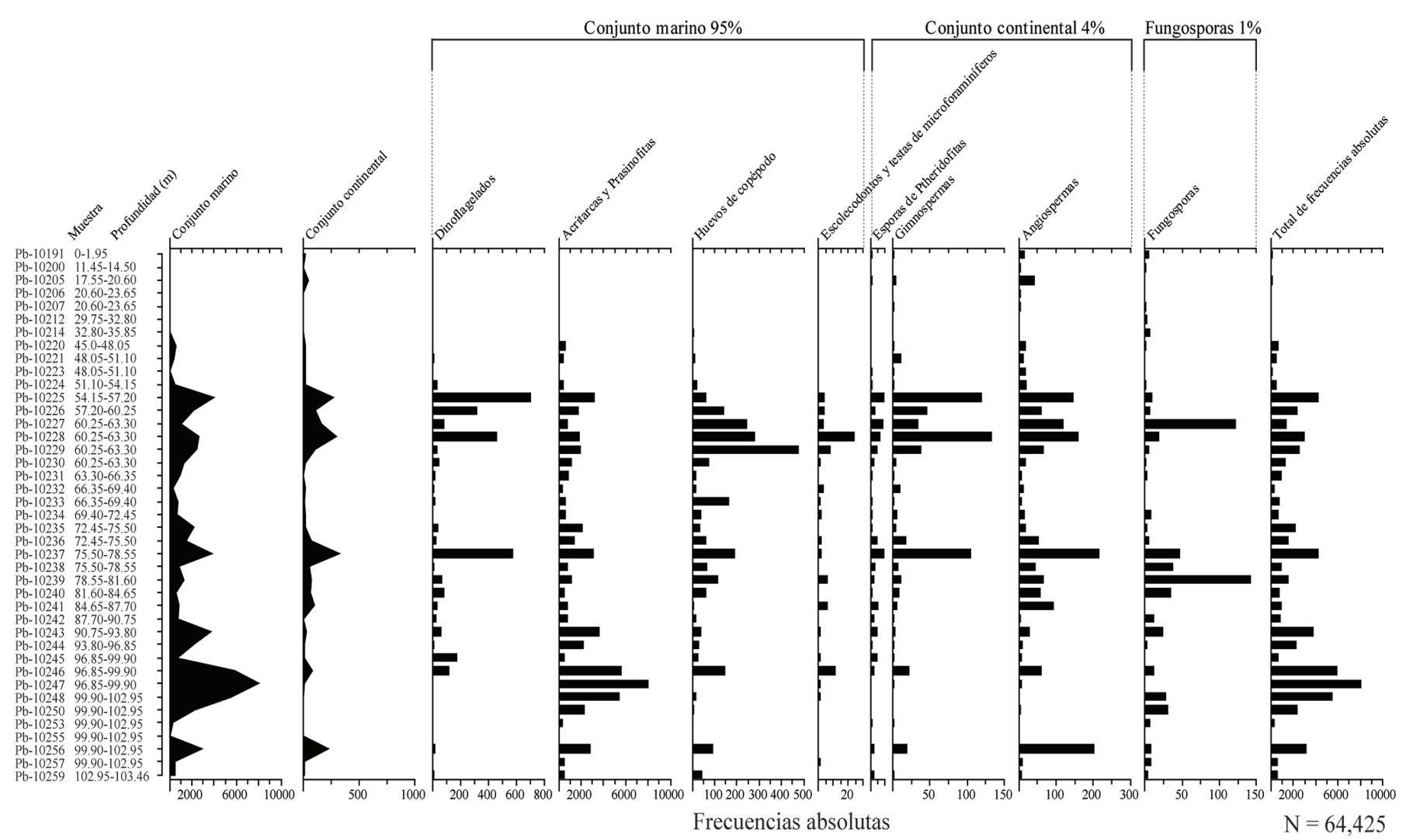

Figura 5 Frecuencias absolutas de los conjuntos continental y marino del barreno 187, San Juan de la Costa, Baja California Sur, México. 
continentales del B187 $\left(H^{\prime}=3.6\right)$ fue sobresaliente en comparación con la del B4 $\left(H^{\prime}=2.61\right)$, mientras que la uniformidad en la distribución de los taxones fue muy similar en los dos barrenos $\left(\mathrm{B} 187, \mathcal{J}^{\prime}=0.6\right.$; B4, $\left.\mathcal{J}^{\prime}=0.51\right)$. En el caso del conjunto de dinoflagelados, los valores de la diversidad a de especies y la uniformidad en el B 187 $\left(H^{\prime}=1.58 ; \mathcal{f}^{\prime}=0.6\right)$ fueron relativamente bajos en comparación con el B4 $\left(H^{\prime}=2.33 ; \mathcal{f}^{\prime}=0.70\right)$.

En ambos barrenos los valores de diversidad indican que la distribución de los individuos dentro de su respectiva especie es relativamente heterogénea, dado que ésta corresponde a una acumulación de especies a través del tiempo. Por otro lado, los valores de la uniformidad $\left(\mathcal{f}^{\prime}\right)$ indican que ambos barrenos son relativamente heterogéneos en dicha distribución.

En general, en el B187 se puede observar una tendencia hacia un registro mayor de palinomorfos continentales y menor de los dinoflagelados. Esto puede ser el resultado de la localización geográfica del B187, cerca de la costa este de Baja California Sur, la cual se encontraba unida al continente durante el Oligoceno superior-Mioceno inferior. Esta condición pudo haber favorecido el aporte de elementos continentales provenientes de tierra adentro, como podría ser el territorio que ocupan actualmente los estados de Sinaloa, Nayarit y Jalisco.

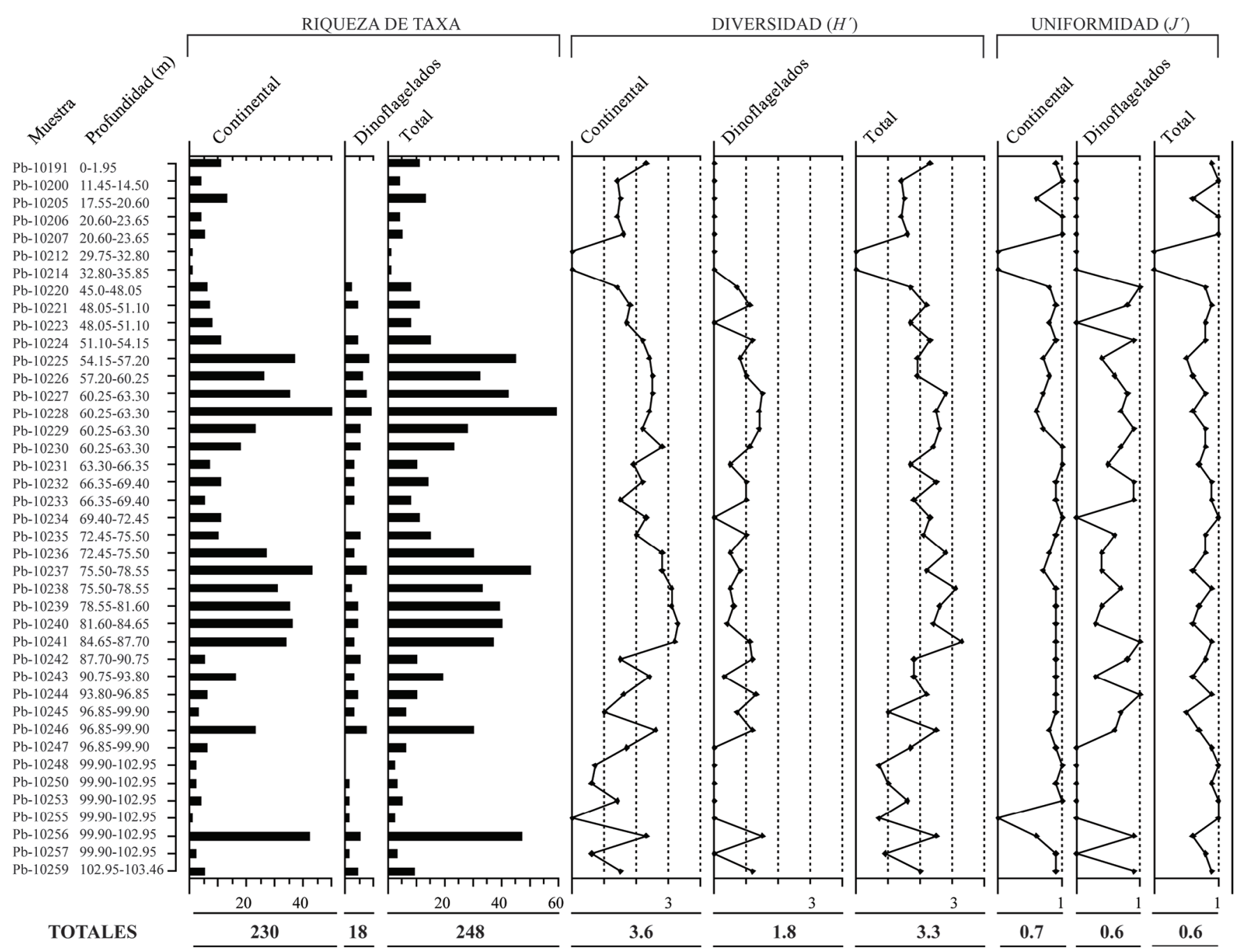

Figura 6 Valores del análisis de diversidad de especies de los conjuntos continental, dinoflagelados y total del barreno 187, San Juan de la Costa, Baja California Sur. 


\subsection{DISTRIBUGIÓN DE TAXONES Y PALINOZONAS EN LA GOLUMNA ESTRATIGRÁFIGA}

La distribución de los taxones, de acuerdo a las profundidades de las muestras en la columna estratigráfica del B187, siguió un patrón que fue identificado claramente por el análisis CONISS. Dicho patrón se caracterizó por una presencia moderada de palinomorfos continentales y dinoflagelados en la base del barreno (Palinozona $\mathrm{A}=\mathrm{Pb}-10241$ a Pb-10259); una concentración máxima de riqueza y abundancia de palinomorfos en la parte media del barreno (Palinozona $\mathrm{B}=$ $\mathrm{Pb}-10220$ a $\mathrm{Pb}-10240)$; y una pobre representación de taxones hacia la cima (Palinozona $\mathrm{C}=$ $\mathrm{Pb}-10191-\mathrm{Pb}-10214)$.

Esta variación en la distribución de los taxones en la columna estratigráfica puede explicarse por la influencia de diversos factores relacionados con la producción y dispersión del polen y las esporas de cada especie, así como con aspectos de sedimentación y tafonomía de los palinomorfos tanto continentales como marinos (Traverse, 2008). La cantidad de palinomorfos continentales en los estratos geológicos está estrechamente relacionada con el aporte eólico, fluvial y la escorrentía; mientras que la cantidad de palinomorfos marinos se vincula con la productividad marina en las zonas costeras y su transporte desde las partes someras de la cuenca a las más profundas (Palafox-Silva, 2013).

\subsection{PALEOVEGETACIÓN}

Algunos de los microfósiles continentales registrados en el análisis del B187 tienen afinidad botánica con familias, géneros o especies vivientes. Con base en las afinidades botánicas es posible inferir los requerimientos ecológicos de cada taxón y, por lo tanto, sugerir los tipos de vegetación en que pudieron desarrollarse.

El análisis de agrupamiento jerárquico por el método de Ward y una distancia euclidiana de 120 destacó tres grupos: A, B1 y B3 (Figura 10). En ellos se concentraron los taxones que dominaron el conjunto palinológico continental. En orden de dominancia se registraron Chenopodipollis y Pinuspollenites, en el grupo A; Graminidites y Eucommia, en el grupo B1; y Retitricolpites, Ephedripites, Psilatricolpites, Fraxinoipollenites, Ephedra claricristatus, Liliacidites, Lusatisporis dettmannae, Tricolporopollenites y el tipo Stercualiaceae, en el grupo B3.

De algunos de éstos se conocen las formas vivientes que son afines a ellos, y la gran mayoría corresponden con elementos que se registran en los estudios florísticos del bosque tropical caducifolio (BTC) (Rzedowski y Calderón de Rzedowski, 1987; Becerra, 2005; Rzedowski, 2006). Dichos taxones fueron: del grupo A, Chenopodipollis que se relaciona con miembros de la familia Amaranthaceae-Chenopodiaceae; y del grupo B1, Graminidites, que se vincula con géneros actuales de la familia Poaceae. En el grupo B3, el BTG está representado por el tipo Sterculiaceae; Ephedripites y Ephedra claricristatus ambas afines al género Ephedra; Liliaciadites que se asocia con géneros de las familias Liliaceae, Bromeliaceae y Palmae; y Psilatricolpites que es afin a miembros de las leguminosas.

Asimismo, en el grupo B2 se registraron bajas frecuencias de una combinación de polen y esporas que se asocian tanto con el BTC como con el bosque mesófilo de montaña (BMM). Entre los elementos que se pueden mencionar, y que están estrechamente relacionados con el BTC, se encontraron Pseudousmodingium, los tipos Labiatae, Meliaceae, Fabaceace, Phytolaccaceae, Anacardiaceae, Leguminosae, Euphorbiaceae, Polygonaceae (Fagopyrum y Rumex), Bursera, Malvacipollis que se vincula con la familia Malvaceae, Tubulifloridites relacionado con Asteraceae, Margocolporites asociado a Caesalpiniaceae y Corsinipollenites que es afin a Onagraceae. Actualmente el BTG es un tipo de vegetación característico de zonas con clima semiseco y cálido ( $\mathrm{Aw}, \mathrm{Bs}$ y $\mathrm{Cw}$ ), en donde las temperaturas promedio anuales van de 20 a $29^{\circ} \mathrm{C}$, con precipitación promedio anual de 300 a $1200 \mathrm{~mm}$ y con una temporada de secas que dura alrededor de cinco a ocho meses, por lo que casi todas las especies pierden sus hojas durante esta temporada (Miranda y Hernández-X., 1963; Rzedowski y Calderón de Rzedowski, 1987; Rzedowski, 2006; Challenger y Soberón, 2008). 


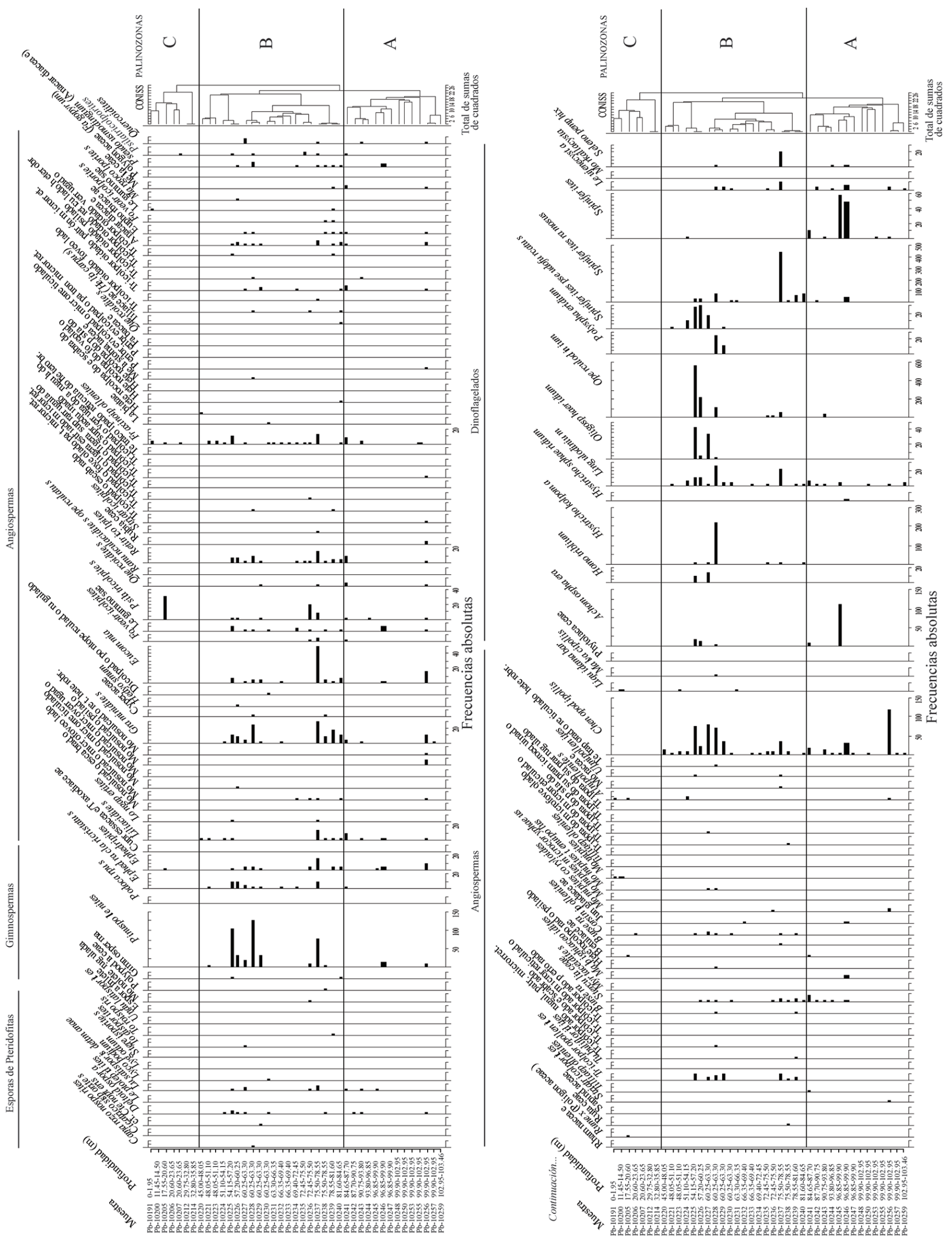

Figura 7 Palinozonas identificadas en la columna estratigráfica del barreno 187, de acuerdo a la distribución de las frecuencias absolutas de los taxones continentales y de dinoflagelados registrados. Formación El Cien, San Juan de la Costa, Baja California Sur, México. 


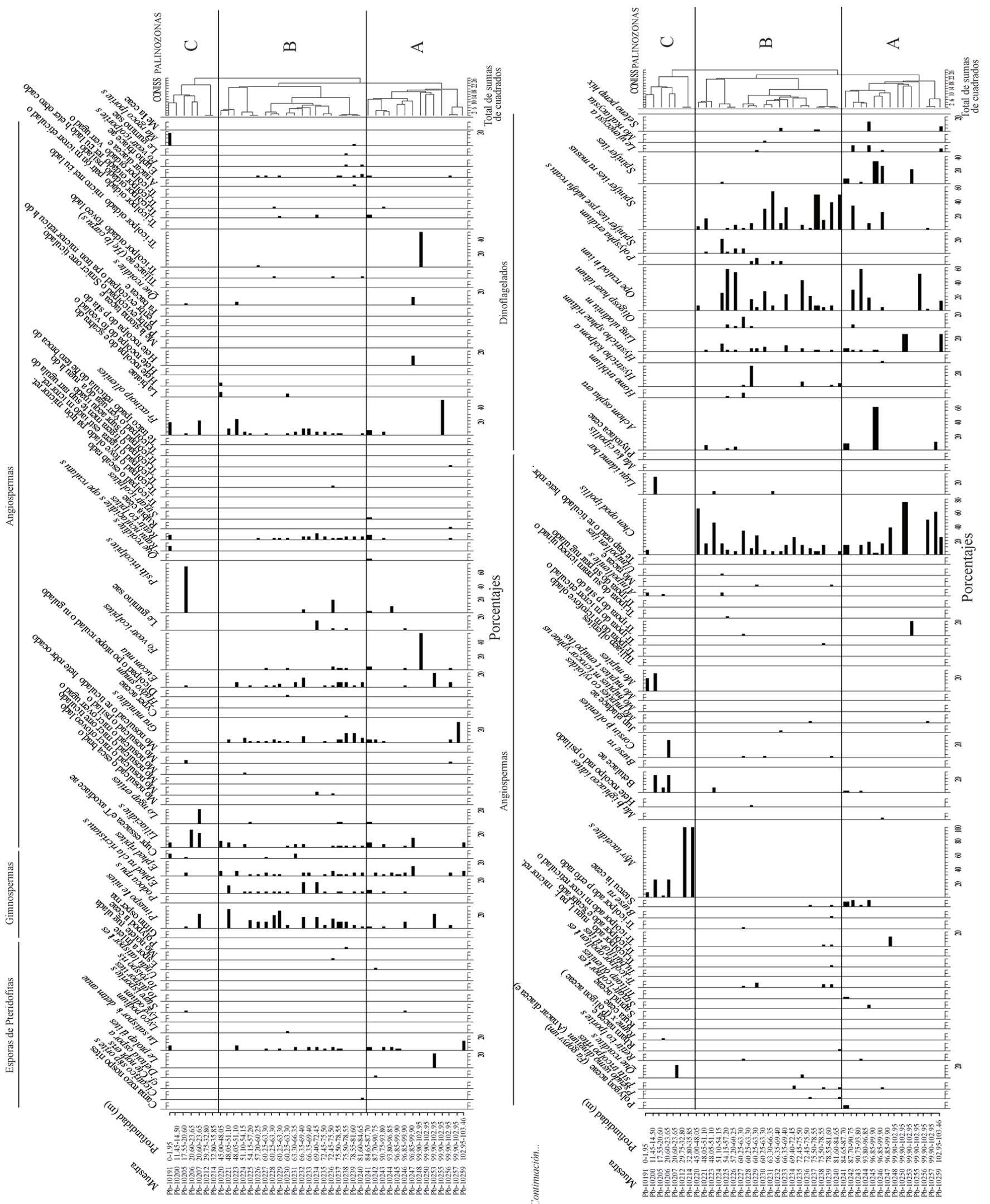

Figura 8 Distribución de los porcentajes del conjunto continental y de dinoflagelados del barreno 187, Formación El Cien, San Juan de la Costa, Baja California Sur, México. 
Por otro lado, el bosque mesófilo de montaña (BMM) estuvo representado por elementos que se encontraron en el grupo Bl como Eucommia; en el B3, Fraxinoipollenites que se relaciona con el género actual de Fraxinus, y Lusatisporis dettmannae, que tiene afinidad botánica con Selaginella oregana (Srivastava, 1972). Cabe destacar que en el grupo B2 se registró la mayor riqueza de elementos que se asocian con el BMM, como los tipos Jungladaceae, Sapindaceae, Rutaceae, Betulaceae, Cupressaceae/Taxodiaceae, Moraceae, Cyperaceae, Melastomataceae, Heliocarpus, Hedyosmum, Podocarpus, Liquidambar y Tilliaepollenites, que se vincula con miembros de la familia Tiliaceae; Momipites, M. tenuipolis, M. microcoryphaeus y $M$. coryloides, que se relacionan con los géneros Engelhardtia, Alfaroa y Oreomunea; Alnipollenites, asociado con Alnus; Myrtaceidites, que tiene afinidad con la familia Myrtaceae; Ulmipollenites con Ulmaceae; así como las esporas de Cicatricosisporites, relacionadas con los géneros Anemia y Mohria; Deltoidospora, asociado a Cyatheaceae, Dicksoniaceae y Schizaeaceae; Undulatisporites, vinculado con Ophioglossaceae, Polypodiaceae, Lygodium, cf. Ctenopteris y Stereisporites, que es un género fósil que incluye esporas de briofitas.

El BMM crece en zonas con un clima templado y húmedo (Cf, Af, Am, Aw y Cw) (Rzedowski, 2006; Challenger y Soberón, 2008), con temperatura media anual que varía de 12 a $23^{\circ} \mathrm{C}$, donde la precipitación es de 1500 hasta 3000 mm anuales, y con frecuentes neblinas y alta humedad atmosférica (Rzedowski, 2006; López-Mata et al., 2012). Se le puede encontrar en altitudes que van desde los 400 hasta los 2700 m (Rzedowski, 2006), pero están mejor representados entre los 1500 y 2500 m (Challenger y Soberón, 2008; López-Mata et al., 2012).

Los dos tipos de vegetación (BTG y BMM) que representaron los palinomorfos continentales del B187 se desarrollan actualmente en diferentes zonas México. El BTC se distribuye principalmente en la vertiente pacífica de México, desde Sonora hasta Yucatán, en el Bajío, en la cuenca del Balsas y en la cuenca del Papaloapan (Miranda y Hernández-X., 1963; Rzedowski y Calderón de Rzedowski, 1987; Rzedowski, 2006; Challenger y Soberón, 2008). Por otro lado, el BMM tiene una distribución limitada y fragmentaria, pero de acuerdo con Rzedowski (1996), pudo haber tenido una distribución más amplia en el Paleógeno-Neógeno, por lo que los manchones que se localizan en los estados de Oaxaca, Chiapas e Hidalgo (Álvarez-Zuñiga et al., 2012), así como en Tamaulipas, San Luis Potosí, Puebla, Veracruz, Sinaloa, Nayarit, Jalisco, Michoacán y Guerrero (Rzedowski, 2006; Baab et al., 2010) son considerados como relictos de este tipo de bosque.

Actualmente en Baja California Sur no existe registro del BMM. Este no es el caso del BTC. Este último se encuentra en las partes inferiores y medias de las sierras de La Laguna y La Giganta (Rzedowski, 2006; León de la Luz et al., 2012). Los registros paleopalinológicos en varias localidades del centro de México (Martínez-Hernández y Ramírez-Arriaga, 1999; Ramírez-Arriaga, 2005; Martínez-Hernández y Ramírez-Arriaga, 2006), así como los estudios sobre el origen, diversificación y cambios espaciales, particularmente del género Bursera (Becerra, 2005), indican la presencia del BTC durante el Paleógeno-Neógeno, desde hace aproximadamente 30 a 20 millones de años, principalmente en el oeste del país, incluyendo a la península de Baja California, de donde posteriormente se expandió hacia el sur y oeste del territorio.

\section{Conclusiones}

Los estratos geológicos del B187 de la Formación El Cien, en la zona de San Juan de la Costa, fueron depositados durante el Oligoceno superior. Dicho barreno fue altamente productivo desde el punto de vista palinológico, registró palinomorfos de origen continental y marino. Aunque los microfósiles marinos dominaron el total del conjunto paleopalinológico, los microfósiles continentales presentaron los valores más altos de riqueza y diversidad de especies. 


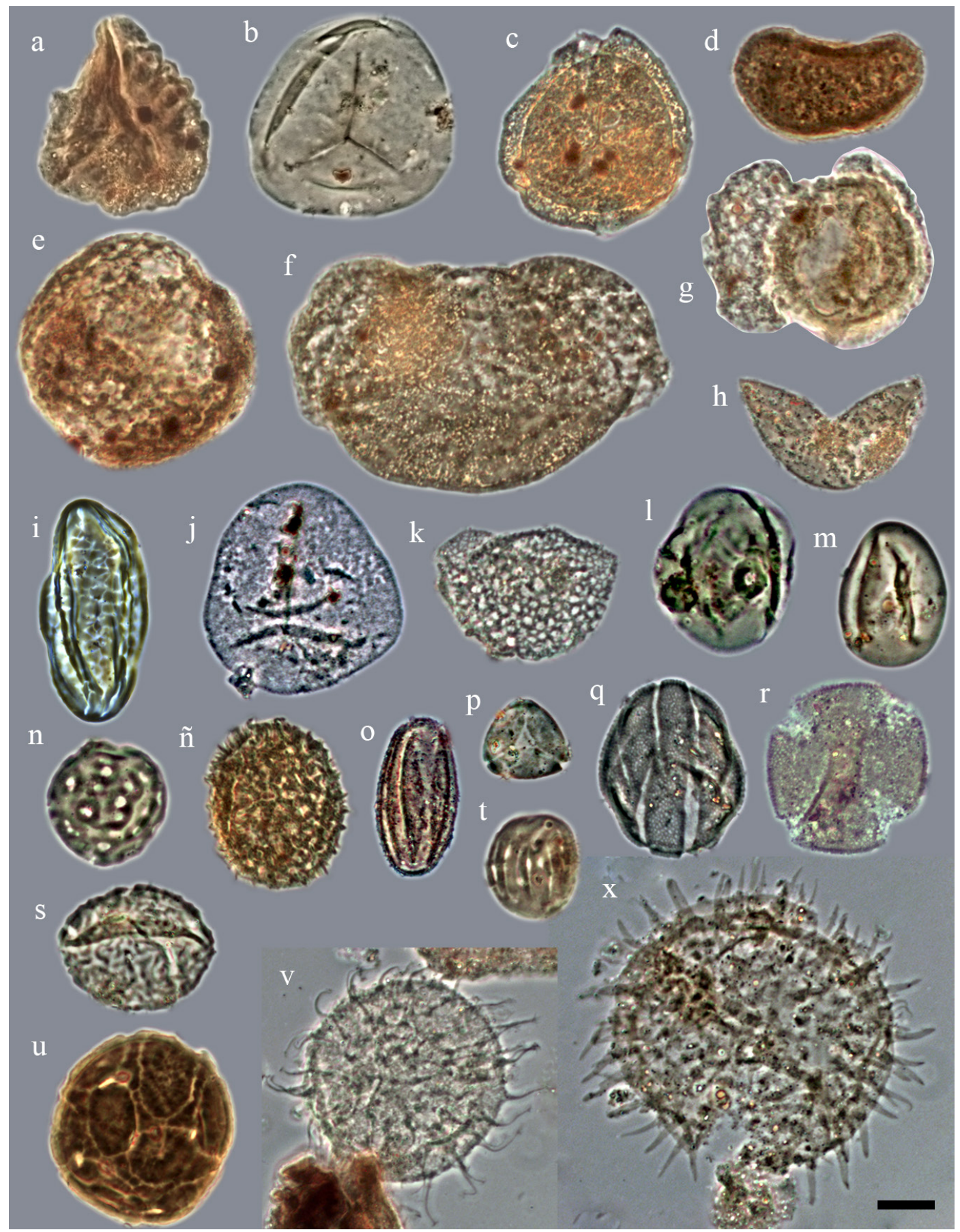

Figura 9 Polen, esporas y dinoflagelados encontrados en el barreno B187, Formación El Cien, San Juan de la Costa, Baja California Sur. La barra de escala representa $10 \mu \mathrm{m}$. Taxones (número de muestra: coordenadas). a) Cicatricosisporites (Pb-10228: 105.5/13.2); b) Deltoidospora (Pb-10230: 105.4/5.2); c) Lusatisporis dettmannae (Pb-10236:111.7/5.6); d) Polypodiacidites (Pb-10238: 110.2/14.4); e) Gimnosperma (Pb-10225:101.2/4.4); f) Pinuspollenites (Pb-10237: 96.4/5.8); g) Podocarpites (Pb-10229: 102.4/15.5); h) Cupressacites/ Taxodiacidites (Pb-10191: 97.9/1 1.4); i) Ephedra claricristatus (Pb-10229: 90.5/3.2); j) Cyperacidites (Pb-10228: 104.9/16.3); k) Liliacidites (Pb-10236: 104.6/12.6); I) Graminidites (Pb-10257: 98.9/5.2); m) Eucommiadites (Pb-10229: 85.4/9.6); n) Chenopodipollis (Pb-10247: 83.7/8.7); ñ) Phytolaccaceae (Pb-10225: 104.4/22.5); o) Psilatricolpites (Pb-10205: 102.3/7.2); p) Myrtaceidites (Pb-10212: 90.0/4.4); q) tipo Labiatae (Pb-10220: 101.6/9.3); r) Fraxinoipollenites (Pb-10241: 98.3/19.4); s) Ulmipollenites (Pb-10237: 101.4/21.6); t) Heterocolpado psilado (Pb-10228:100.8/8.2); u) Malpighiaceoidites (Pb-10229: 93.1/3.4); v) Operculodinium (Pb-10225: 102.7/7.3); x) Lingulodinium (Pb-10225: 90.9/8.7). 


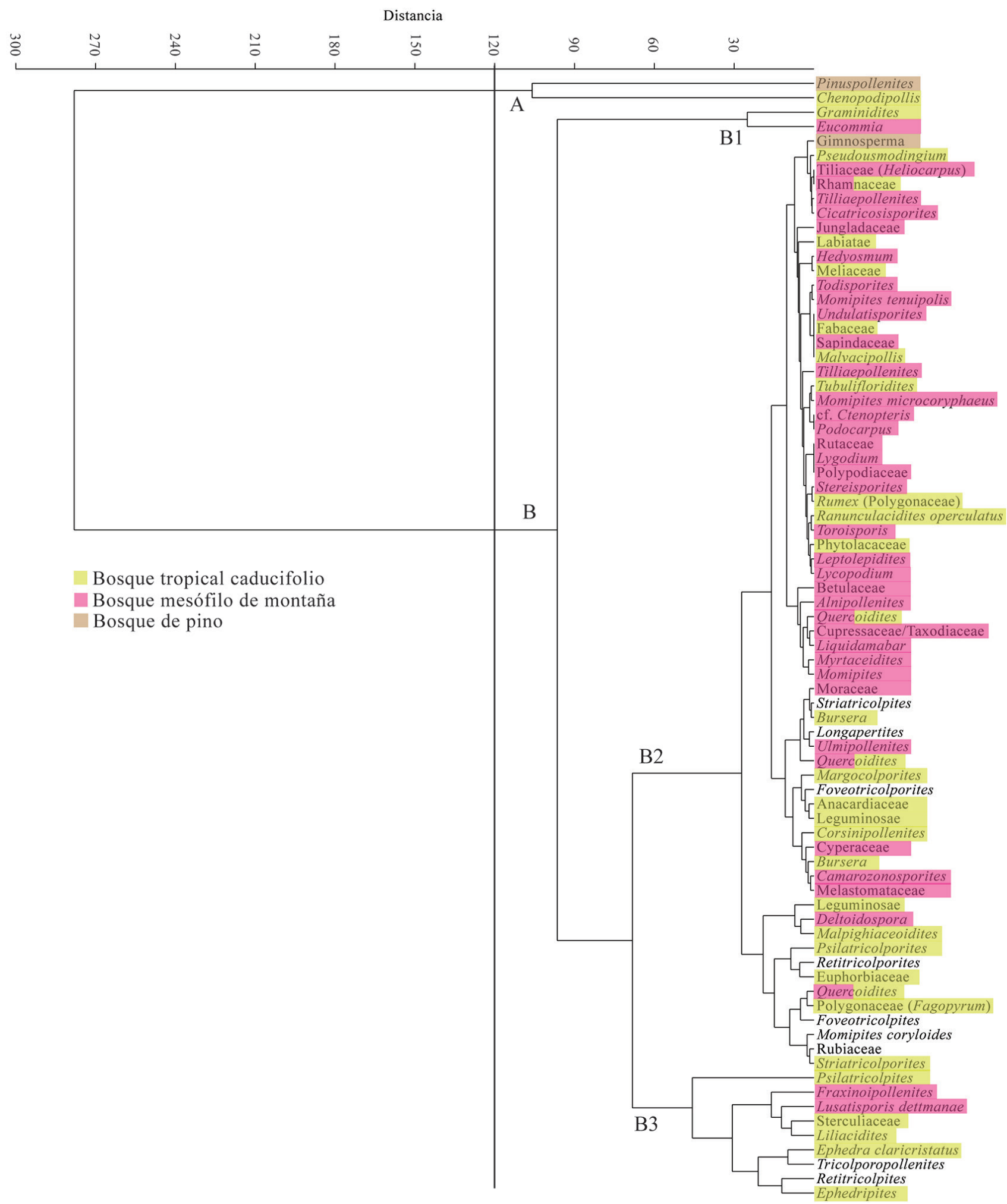

Figura 10 Dendrograma del análisis de agrupamiento jerárquico para observar los tipos de vegetación representados en el barreno 187, Formación El Cien, San Juan de la Costa, Baja California Sur, México. 
En la columna estratigráfica del B187 se lograron distinguir tres palinozonas de acuerdo a la profundidad de cada muestra obtenida y a la distribución de los taxones en éstas. La palinozona B presentó la mayor riqueza y diversidad de especies de elementos continentales y de dinoflagelados, mientras que las palinozonas A y $\mathrm{C}$ se caracterizaron por la escasez de polen, esporas y dinoflagelados.

Los taxones que dominaron el espectro del conjunto continental fueron: Chenopodipollis (Amaranthaceae-Chenopodiaceae), Pinuspollenites (Pinus), Graminidites (Poaceae) y Eucommia, Retitricolpites, Ephedripites (Ephedra), Psilatricolpites (Leguminosae), Fraxinoipollenites (Fraxinus), Ephedra claricristatus (Ephedra), Liliacidites (Liliaceae, Bromeliaceae y Palmae), Lusatisporis dettmannae (Selaginella oregana), Tricolporopollenites y el tipo Stercualiaceae. En contraparte, en el conjunto de dinoflagelados destacaron por su abundancia Operculodinium y Spiniferites ramosus.

Los taxones representados en el conjunto continental permitieron identificar dos tipos de vegetación predominantes durante el Oligoceno superior en los alrededores de San Juan de la Costa: el bosque tropical caducifolio y el bosque mesófilo de montaña. Los datos sugieren que el bosque tropical caducifolio se desarrollaba en las cercanías a la zona de depósito del B187; mientras que el bosque mesófilo de montaña pudo estar presente en las partes altas y húmedas de los alrededores de la zona de contacto de la península de Baja California con los estados de Sinaloa, Nayarit y Jalisco, cuando la península se encontraba unida al continente (hace 27.5 Ma).

\section{Agradecimientos}

Al Proyecto PAPIIT-IN114914-3-DGAPAUNAM. Al CONACyT, por la beca de posgrado asignada a la primera autora. Al personal de ROFOMEX: Ingenieros Carlos Salcido, Moisés Martínez, Rodrigo Diez y William González, por la donación del barreno B187, por la información proporcionada y por su generosa atención y ayuda dentro de su empresa y en los recorridos de campo en la zona de San Juan de la Costa, BCS.
La revisión crítica de la Dra. María del Carmen Zamaloa y el Dr. Luis Palazzesi, mejoraron sustancialmente el manuscrito. Al Dr. Lauro López Mata, Dr. Enrique Martínez Hernández y Dr. Javier Helenes Escamilla por sus valiosos comentarios y sugerencias al manuscrito. Al personal del laboratorio de paleopalinología del IG-UNAM y del Posgrado en Botánica del Colegio de Postgraduados, por las facilidades otorgadas para realización de este trabajo.

\section{Referencias}

Alatorre, A.E., 1988, Stratigraphy and depositional environments of the phosphorite-bearing Monterrey Formation in Baja California Sur: Economic Geology, 83(8), 1918-1930. https:// doi.org/10.2113/gsecongeo.83.8.1918

Álvarez-Zuñiga, E., Sánchez-González, A., López-Mata, L., Tejero-Diez, J.D., 2012, Composición y abundancia de las pteridofitas en el bosque mesófilo de montaña del municipio de Tlanchinol, Hidalgo, México: Botanical Sciences, 90(2), 163-177. https:// doi.org/10.17129/botsci.481

Applegate, S.P., 1986, The El Cien Formation: Strata of Oligocene and early Miocene age in Baja California Sur: Universidad Nacional Autónoma de México, Instituto de Geología, Revista, 6(2), 145-162.

Aranda-Gómez, JJ., Henry, C.D., Luhr, J.F., 2000, Evolución tectonomagmática post-paleocénica de la Sierra Madre Occidental y de la porción meridional de la provincia tectónica de Cuencas y Sierras, México: Boletín de la Sociedad Geológica Mexicana, 53(1), 59-71. https://doi. org/10.18268/bsgm2000v53nla3

Baab, K., Blanco y Correa, M., González Flores, B.E., Navarrete Carrillo, J.A., Téllez Valdés, O., Vega-Aviña, R., 2010, Serranías de Nayarit, en Toledo-Aceves, T. (ed.), El bosque mesófilo de montaña en México: Amenazas y oportunidades para su conservación y manejo sostenible: México, Comisión Nacional para el conocimiento y uso de la biodiversidad, 50-59. 
Becerra, J.X., 2005, Timing the origin and expansion of the Mexican tropical dry forest (resumen) in Proceedings of the National Academy of Sciences of the United States of America: United States of America, National Academy of Sciences of the United States of America, 102(31), 10919-10923. https://doi. org/10.1073/pnas.0409127102

Carreño, A.L., Helenes,J., 2002, Geology an Ages of the Islands, in Case, T.J., Cody, M. L., Ezcurra, E. (eds.), A new Island Biogeography of the Sea of Cortes: New York, U.S.A., Oxford University Press, 14-40

Carreño, A.L., Smith, J.T., 2007, Stratigraphy and correlation for the ancient Gulf of California and Baja California Peninsula, México: Bulletin of American Paleontology, 37 1, 1-146.

Challenger, A., Soberón, J., 2008, Los Ecosistemas Terrestres, en Soberón, J., Halffter, G., LlorenteBosques, J. (eds.), Capital Natural de México: Conocimiento actual de la diversidad, Vol.I: México, CONABIO, 87-108.

Fischer, R., Galli-Olivier, C., Gidde, A., Schwennicke, T., 1995, The El Cien Formation of southern Baja California, Mexico: Stratigraphic precisions, Newsletters on Stratigraphy, 32(3), 137-161. https://doi.org/10.1127/ nos/32/1995/137

Galván-Escobedo, I.G., Ramírez-Arriaga, E., Uscanga-Mortera, E., Valiente-Banuet, A., García-Moya, E., Kohashi-Shibata, J., Martínez-Hernández, E., 2012， Registro Paleopalinológico Preliminar del Barreno B4 de la Formación San Gregorio en Comondú, Baja California Sur, México (resumen en extenso), en Memorias de la Convención Nacional Geológica 2012 [En línea]: World Trade Center, Ciudad de México, Sociedad Geológica Mexicana, A.C. http://sociedadgeologica.org. mx/galeria_convencion_2012.html [Fecha de consulta: 26 de mayo de 2013].

Galván-Escobedo, I.G., Ramírez-Arriaga, E., Valiente-Banuet, A., Helenes-Escamilla, J., 2013, Paleopalynological comparison of San Gregorio Formation and El Cien Formation, Baja California Sur, Mexico: a preliminary analysis (resumen), in AASP-Tps 46th Annual
Meeting, San Francisco, California, U.S.A., American Association of Stratigraphic Palynologists (AASP), 88-89.

Galván-Escobedo, I.G., Ramírez-Arriaga, E., ReyesSalas, M., Martínez-Hernández, E., ÁngelesGarcía, S., García-Moya, E., 2015, Descriptions and botanical affinities of Tubulifloridites Cookson 1947 ex Potonié 1960 pollen grains from the San Gregorio Formation, Baja California Sur, Mexico: Acta Microscópica, 24(1), 7-15. http://actamicroscopica.ivic. gob.ve/V24_1_2015/Art\%C3\%ADculos_ V240115/240115-07.pdf

Galván-Escobedo, I.G., Ramírez-Arriaga, E., Valiente-Banuet, A., Uscanga-Mortera, E., García-Moya, E., Kohashi-Shibata, J., 2017, Registro paleopalinológico de la formación San Gregorio (Barreno B4), Baja California Sur, México: Boletín de la Sociedad Geológica Mexicana, 69(1), 35-58. https://doi. org/10.18268/bsgm2017v69nla3

Grimm, E.G., 1987, CONISS: a FORTRAN 77 program for stratigraphically constrained cluster analysis by the method of incremental sum of squares: Computer \& Geosciences, 13(1), 13-35. https://doi. org/10.1016/0098-3004(87)90022-7

Grimm, E.C., 2011, Tilia Software. Versión 1.7.16, ed. Springfield, IL.: Illinois State Museum.

Hausback, B.P., 1984, Cenozoic Volcanic and Tectonic Evolution of Baja California Sur, México, in Frizzell, I. (ed.), Geology of the Baja California Peninsula: Los Angeles, California, U.S.A., Pacific Section, Society of Economic Paleontologists and Mineralogists, 219-236.

Johnson, D.E., 2000, Métodos multivariados aplicados al análisis de datos: México, International Thomson Editores, 566 p.

Kim, W.H., Barron, J.A., 1986, Diatom biostratigraphy of the upper Oligocene to lowermost Miocene San Gregorio Formation, Baja California Sur, Mexico: Diatom Research, 1(2), 169-187. https://doi. org/10.1080/0269249x.1986.9704967

Ledesma-Vázquez, J., Carreño, A.L., 2010, Origin, age, and geological evolution of the Gulf of California, 
in Brusca, R. G. (ed.), The Gulf of California. Biodiversity and conservation: Arizona, U.S.A., The University of Arizona Press and The ArizonaSonora Desert Museum, 7-23.

León de la Luz, J.L., Domínguez-Cadena, R., Mendel-Narváez, N., 2012, Florística de la Selva Baja Caducifolia de la Península de Baja California, México: Botanical Sciences, 90(2), 143-162. https://doi.org/10.17129/ botsci. 480

López-Mata, L., Villaseñor,J.L., Cruz-Cárdenas, G., Ortíz, E., Ortíz-Solorio, C., 2012, Predictores ambientales de la riqueza de especies de plantas del Bosque Húmedo de Montaña de México: Botanical Sciences, 90(1), 27-36. https://doi.org/10.17129/botsci.383

Magurran, A.E., 1989, Diversidad ecológica y su medición: Barcelona, España, Vedra, 200 p.

Martínez-Hernández, E., Ramírez-Arriaga, E., 1999, Palinoestratigrafía de la región de Tepexi de Rodríguez, Puebla, México. Implicaciones Cronoestratigráficas: Revista Mexicana de Ciencias Geológicas, 16(2), 187-207. https://www.uv.mx/personal/tcarmona/ files /2010/08/Martinez-y-Ramirez-1999. pdf

Martínez-Hernández, E., Ramírez-Arriaga, E., 2006, Tertiary Palynofloristic correlations between Mexican Formations with emphasis in dating the Balsas Group, in Vega, F., Nyborg, T. G., Perrilliat, M. C., Montellano-Ballesteros, M., Cevallos-Ferriz, S., Quiroz-Barroso, S.A. (eds.), Studies on Mexican Paleontology: Dordrecht, The Netherlands, Springer, 19-45. https://doi. org/10.1007/1-4020-3985-9_2

Miranda, F., Hernández-X., E., 1963, Los tipos de vegetación de México y su clasificación: Boletín de la Sociedad Botánica de México, 28, 29-179. http://dx.doi.org/10.17129/ botsci. 1084

Palafox-Silva, L.H., 2013, Registro palinológico en sedimentos laminados de la cuenca La Paz, Baja California Sur y su relación con cambios paleoceanográficos y paleoclimáticos: Ensenada, BC, México,
Centro de Investigación Científica y de Educación Superior de Ensenada, Tesis de maestría, $80 \mathrm{p}$.

Pielou, E.C, 1975, Ecological Diversity: New York, Wiley InterScience, 165 p.

Ramírez-Arriaga, E., 2005, Reconstrucción Paleoflorística de la Formación Guayuca con base en análisis palinoestratigráfico e implicaciones paleogeográficas: México, Universidad Nacional Autónoma de México, Tesis doctoral, $231 \mathrm{p}$.

Rzedowski, J., 1996, Análisis preliminar de la flora vascular de los bosques mesófilos de montaña de México: Acta Botánica Mexicana, 35, 25-44. https://doi.org/10.21829/ abm35.1996.955

Rzedowski,J. 2006, Vegetación de México: México, Comisión Nacional para el Conocimiento y Uso de la Biodiversidad, 504 p.

Rzedowski, J., Calderón de Rzedowski, G., 1987, El bosque tropical caducifolio de la región mexicana del Bajío: Trace, 12, 12-21.

Seaby, R.M.H., Henderson, P.A., 2007, Species diversity and richness IV: Lymington, Hampshire, Pisces Conservation Ltd., 132 p.

Smith, J.T., 1991, Genozoic marine mMollusks and paleogeography of the Gulf of California, in Dauphin, J.P., Simoneit, B.R.T. (eds.), The Gulf and peninsular province of the Californias: Oklahoma, USA, American Association of Petroleum Geologists in Tulsa, 637-666.

Solari, L.A., Gómez-Tuena, A., Bernal, J.P., Pérez-Arvizu, O., Tanner, M., 2010, $\mathrm{U}-\mathrm{Pb}$ zircon geochronology with an integrated LA-ICP-MS microanalytical workstation: Achievements in Precision and Accuracy: Geostandards and Geoanalytical Research, 34, 5-18. https://doi. org/10.1111/j.1751-908x.2009.00027.x

Srivastava, S.K., 1972, Systematic description of some spores from The Edmonton Formation Maestrichtian), Alberta, Canada: Palaeontographica, B, 139, 1-46.

Traverse, A., 2008, Paleopalynology: Netherlands, Springer, $813 \mathrm{p}$. 